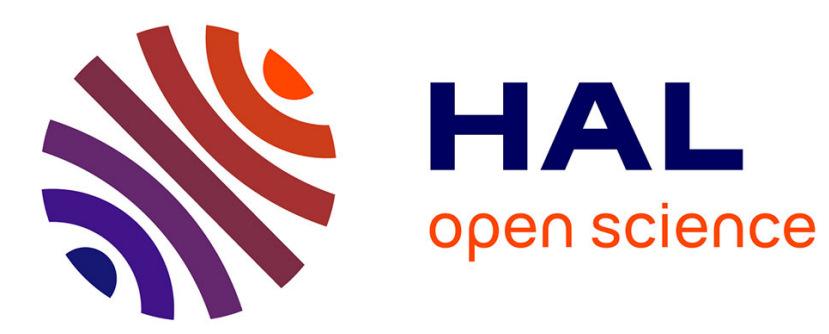

\title{
La nouvelle veine d'essais du tunnel hydrodynamique du LEGI
}

Muriel Lagauzere, Jean-Pierre Franc

\section{To cite this version:}

Muriel Lagauzere, Jean-Pierre Franc. La nouvelle veine d'essais du tunnel hydrodynamique du LEGI. La Houille Blanche - Revue internationale de l'eau, 2018, 4, pp.73 - $76.10 .1051 / \mathrm{lhb} / 2018044$. hal01905662

\section{HAL Id: hal-01905662 \\ https://hal.science/hal-01905662}

Submitted on 27 Nov 2018

HAL is a multi-disciplinary open access archive for the deposit and dissemination of scientific research documents, whether they are published or not. The documents may come from teaching and research institutions in France or abroad, or from public or private research centers.
L'archive ouverte pluridisciplinaire $\mathbf{H A L}$, est destinée au dépôt et à la diffusion de documents scientifiques de niveau recherche, publiés ou non, émanant des établissements d'enseignement et de recherche français ou étrangers, des laboratoires publics ou privés. 


\title{
La nouvelle veine d'essais du tunnel hydrodynamique du LEGI
}

\author{
Muriel LAGAUZERE, Jean Pierre FRANC
}

Univ. Grenoble Alpes, CNRS, Grenoble INP, LEGI, 38000 Grenoble,France, e-mail: Muriel.Lagauzere@legi.cnrs.fr ,Jean-Pierre.Franc@legi.cnrs.fr

\begin{abstract}
RÉSUMÉ. - Cette note présente les principaux choix techniques réalisés à l'occasion de la rénovation récente de l'une des veines d'essais du tunnel hydrodynamique du Laboratoire des Ecoulements Géophysiques et Industriels de Grenoble. Après une brève description du nouveau circuit hydraulique, le dispositif de supervision des conditions de fonctionnement de l'installation est présenté. Le profil à tester, monté dans la veine d'essais, est piloté en incidence par un moteur brushless permettant de lui communiquer une loi quelconque de variation d'incidence en fonction du temps. Ce dispositif offre une grande flexibilité et ouvre des perspectives nouvelles en matière de recherche sur les écoulements cavitants instationnaires. Un système d'acquisition haute fréquence a été également développé afin d'acquérir en simultané les signaux d'une matrice de capteurs de pression PVDF miniatures équipant le profil d'essais. Ce type de mesure est particulièrement adapté à l'évaluation du potentiel érosif d'un écoulement cavitant. L'acquisition des signaux est synchronisée à l'acquisition d'images par vidéo rapide afin de corréler pressions instationnaires et structures de vapeur et permettre ainsi l'interprétation des signaux de pression.
\end{abstract}

Mots-clés : tunnel hydrodynamique, cavitation, supervision, acquisition de données.

\section{The New Test Section of the LEGI Hydrodynamic Tunnel}

\begin{abstract}
This note presents the main technical choices made recently when rehabilitating one of the test sections of the hydrodynamic tunnel of the Laboratory of Geophysical and Industrial Flows (LEGI) in Grenoble. After a brief description of the new design of the hydraulic test section, the acquisition system for monitoring the facility operating point is presented. The hydrofoil to be tested is driven by a brushless electric motor able to move the hydrofoil according to an arbitrary law for the variation of its angle of attack as a function of time. The system offers high flexibility and promising research developments in the field of unsteady cavitating flows. A high frequency acquisition system was also developed for the simultaneous acquisition of an array of miniature PVDF pressure sensors flush mounted of the hydrofoil surface. Such measurements allow estimating the erosive potential of a cavitating flow. Signal acquisition was synchronized with high-speed videos in order to correlate unsteady pressure signals to vapor structure collapses and assist in the interpretation of pressure signals.
\end{abstract}

Key-words: hydrodynamic tunnel, cavitation, monitoring, data acquisition.

\section{INTRODUCTION}

Le tunnel hydrodynamique du LEGI est un grand équipement du laboratoire $(165 \mathrm{~kW}, 750 \mathrm{l} / \mathrm{s})$ permettant de réaliser divers types d'essais hydrauliques et en particulier des essais de cavitation. Depuis sa mise en service en 1967, le tunnel hydrodynamique du LEGI a fait l'objet de plusieurs évolutions majeures. En 1974, une seconde veine d'essais a été construite en parallèle de la première et une pré-cuve aval a été ajoutée à la cuve aval d'origine permettant une meilleure extraction des grandes quantités d'air injectables lors d'essais de ventilation. En 1985-86, le tunnel a été doté d'un ensemble de contrôle de la qualité de l'eau, essentiel aux travaux de recherche sur la cavitation. Cet ensemble comporte un système d'ensemencement en germes de cavitation complété d'un dispositif de mesure de la population en germes de l'eau du tunnel [Briançon-Marjollet et Michel, 1990]. En 2004, des aménagements importants ont été réalisés sur la seconde veine d'essais en vue de la convertir en un banc d'essais de modèles réduits d'hydroliennes [Guittet, Kusulja et Maître, 2004].

La première veine d'essais vient d'être entièrement rénovée afin de répondre aux besoins nouveaux de la recherche en cavitation. Cette note présente les choix techniques qui ont été faits concernant le circuit hydraulique (paragraphe II), la supervision des paramètres de fonctionnement de la veine d'essais (paragraphe III), le système de pilotage en incidence des profils d'essais (paragraphe IV) et le dispositif d'acquisition haute fréquence des mesures fines en veine d'essais (paragraphe V).

\section{DESIGN HYDRAULIQUE DE LA NOUVELLE VEINE D'ESSAIS}

La figure 1 présente une vue d'ensemble de la nouvelle veine d'essais. Elle comporte les éléments suivants. A l'amont, un raccord rond/carré assure la transition entre un coude à ailettes DN800 (non représenté sur la figure) et le caisson d'injection de microbulles de section carrée. Ce caisson est équipé d'injecteurs permettant soit de contrôler la concentration en germes pour des essais de cavitation, soit d'ensemencer l'eau en traceurs non polluants pour des mesures LDV (vélocimétrie laser doppler) ou PIV (vélocimétrie par images de particules). Des informations détaillées sur les dispositifs d'injection de microbulles équipant le tunnel du LEGI et sur leurs performances sont disponibles dans Aumelas et al., 2016, 2018. Ce caisson comporte 


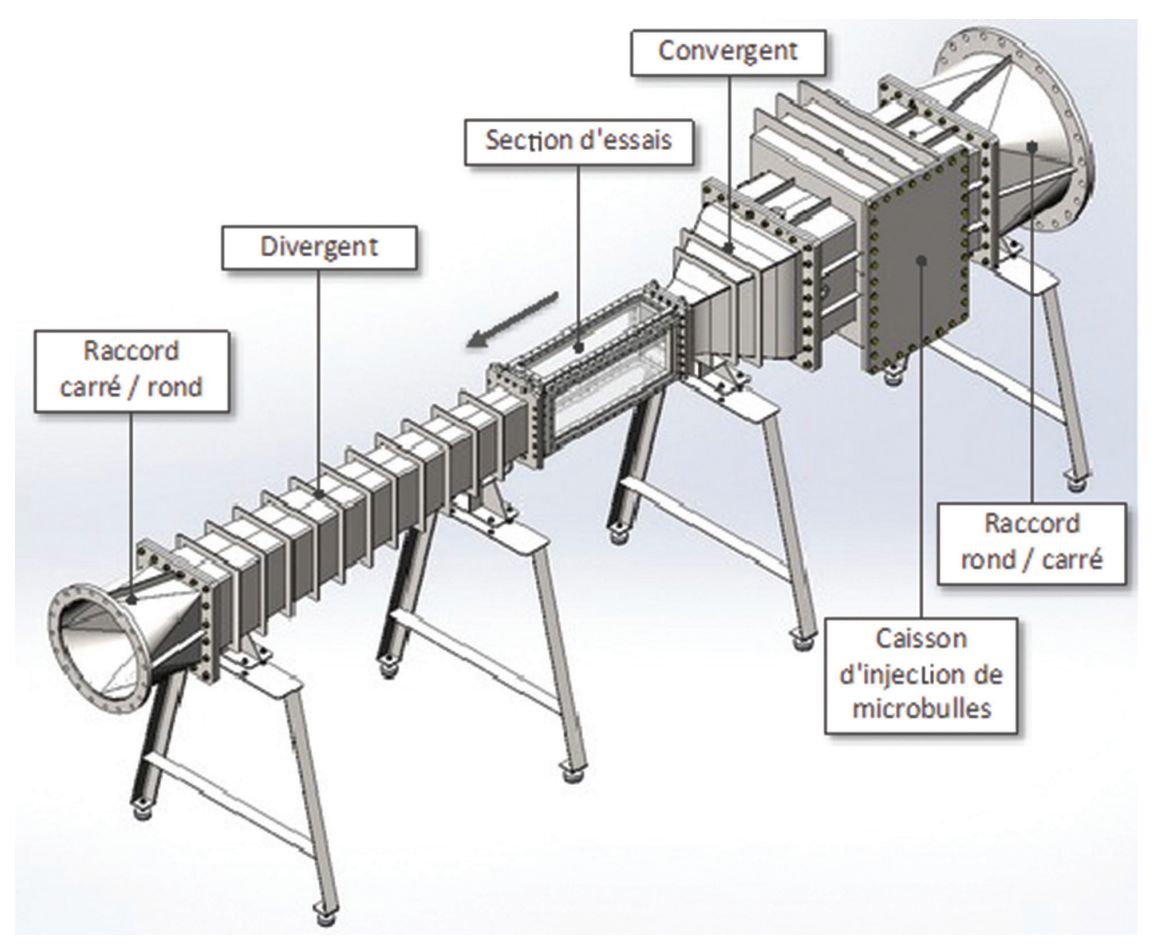

Figure 1: Vue de la nouvelle veine d'essais du tunnel hydrodynamique du LEGI.

également un nid d'abeille assurant une homogénéisation de l'écoulement. Il est suivi d'un convergent permettant le raccordement entre la section carrée de sortie du caisson (560 $\mathrm{mm} \times 560 \mathrm{~mm}$ ) et l'entrée rectangulaire de la veine d'essais. Celle-ci, entièrement transparente et dans laquelle est monté un profil d'essais, a une hauteur de $280 \mathrm{~mm}$, une largeur de $175 \mathrm{~mm}$ et une longueur de $1 \mathrm{~m}$. Elle est suivie d'un divergent dont la divergence est faible afin de limiter les zones de décollement (angle de divergence de 4 degrés horizontalement et 3 degrés verticalement). Le raccordement à la pré-cuve aval (DN 500) se fait grâce à une transition carré/rond. L'écoulement venant de la veine d'essais se jette dans un déversoir intégré à la pré-cuve. Il s'agit d'un canal à surface libre, fermé en bout, qui fonctionne par débordement et qui permet ainsi de maintenir la veine d'essais en charge et d'éviter qu'elle ne se dénoie, quel que soit le point de fonctionnement de l'installation.

\section{LA SUPERVISION DES PARAMÈTRES DE LA VEINE DE CAVITATION}

La supervision des paramètres de fonctionnement de la veine de cavitation se fait par l'intermédiaire du module d'acquisition National Instruments USB 6343. Ce module permet l'acquisition de 32 entrées analogiques $( \pm 10 \mathrm{~V})$ avec une résolution de 16 bits. La fréquence d'acquisition de la carte peut monter jusqu'à $500 \mathrm{kHz}$ si une seule voie est utilisée. Dans le cas de l'utilisation de plusieurs voies d'acquisition, la fréquence est divisée par le nombre de voies.

Le logiciel d'acquisition et de contrôle commande LabVIEW est utilisé pour acquérir en continu les signaux des capteurs de pression amont, de température et de débit à une fréquence de $1000 \mathrm{~Hz}$ par voie. La figure 2 présente la face avant du programme de supervision des paramètres de la veine de cavitation. Les signaux en courant sont convertis en tension par l'ajout d'une résistance de précision sur la carte d'acquisition. Par l'intégration de boites de calcul dans le code, les valeurs en tension sont converties en valeurs physiques. Nous accédons ainsi aux valeurs de pression amont (bar), de température de l'eau $\left({ }^{\circ} \mathrm{C}\right)$ et de débit $(1 / \mathrm{s})$. En renseignant les caractéristiques de la veine d'essai dans le programme et en tenant compte des données d'acquisition, nous obtenons par calcul les valeurs du nombre de cavitation, du nombre de Reynolds, de la vitesse d'entrée dans la veine d'essai $(\mathrm{m} / \mathrm{s})$ mais aussi de la pression d'entrée dans la veine $(\mathrm{Pa})$ et de la pression dans la cuve aval (bar). Ces valeurs sont moyennées et affichées sous forme de graphes déroulants et d'indicateurs numériques au cours de l'acquisition. Elles permettent d'obtenir une supervision du point de fonctionnement de la veine d'essais.

L'utilisateur a la possibilité de procéder à un enregistrement des données dans un fichier de mesures. Le nom de ce fichier est étiqueté avec les valeurs mesurées afin d'identifier aisément les conditions d'essais. Dans ce fichier de mesures sont stockées les valeurs en tension mais également l'ensemble des valeurs physiques citées ci-dessus. Le déclenchement de l'enregistrement du fichier de mesures peut être synchronisé avec la prise éventuelle d'images sur la veine de cavitation par une caméra rapide Miro. La prise d'images se termine automatiquement en même temps que l'enregistrement du fichier de mesures. L'utilisateur a la possibilité d'arrêter à tout moment le programme de supervision de la veine de cavitation par action sur un bouton stop.

\section{LE SYSTÈME DE PILOTAGE DES PROFILS}

La figure 3 présente un profil typique monté dans le tunnel. La mise en incidence du profil va mettre le liquide en dépression et créer de la cavitation. L'extrados du profil est 


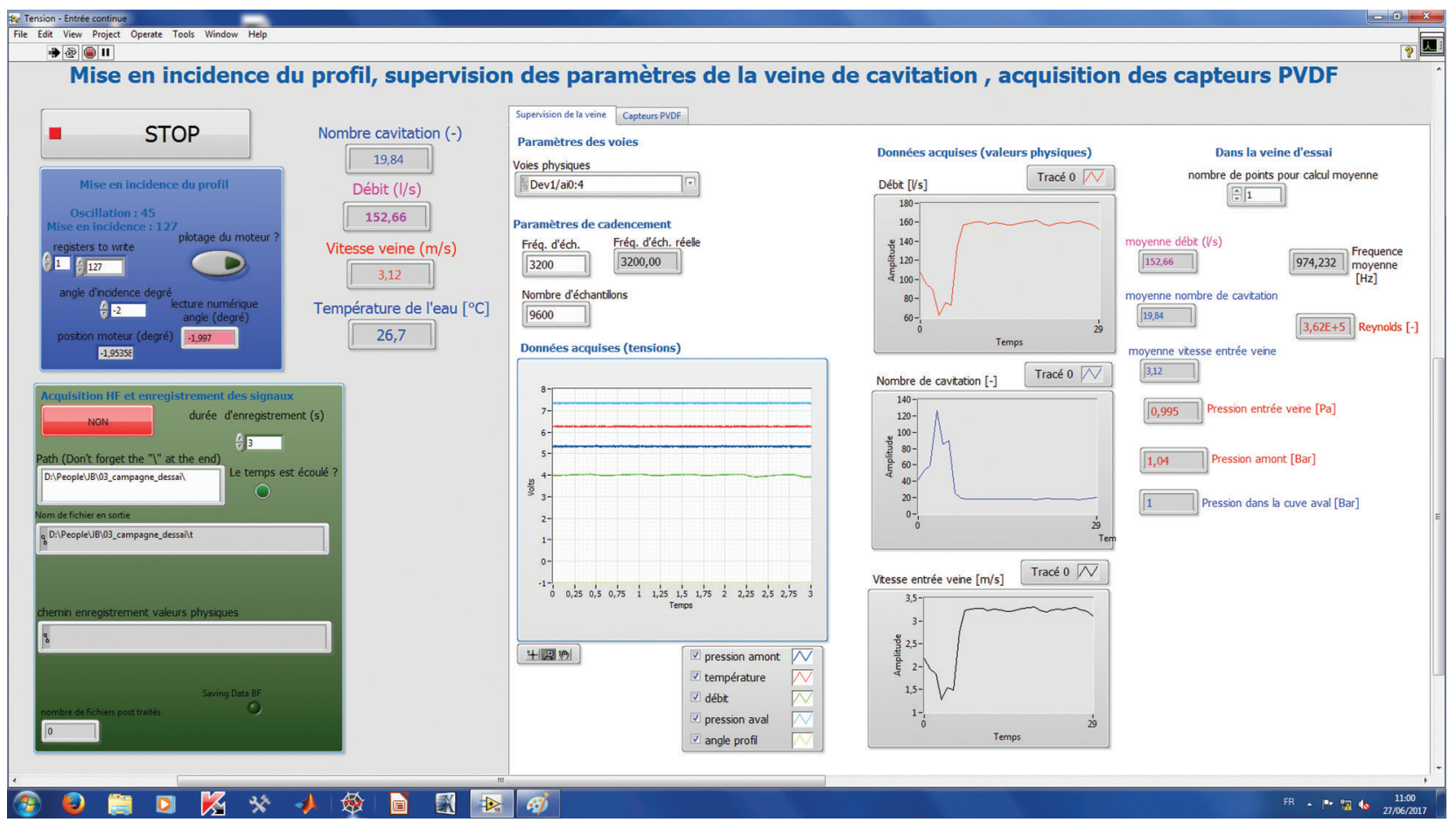

Figure 2: Face avant du programme de supervision de la veine de cavitation.

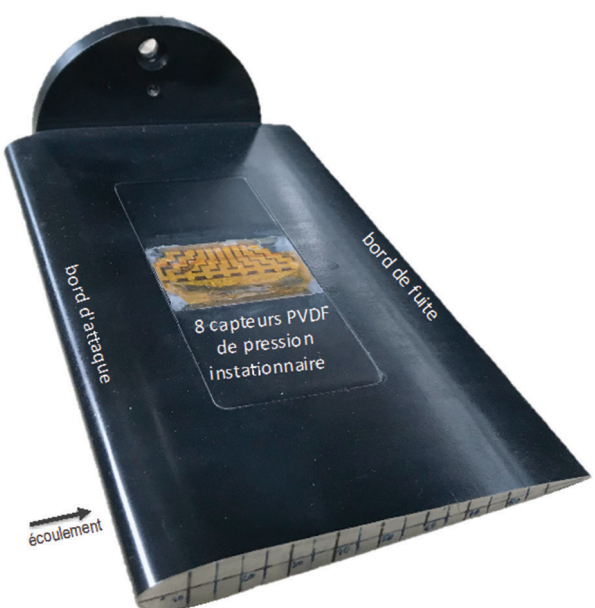

Figure 3 : Exemple de profil instrumenté.

instrumenté à l'aide de huit capteurs de pression instationnaire réalisés au laboratoire à partir de film piézoélectrique de polyfluorure de vinylidène (PVDF) d'épaisseur $40 \mu \mathrm{m}$. Ce type de capteur est particulièrement adapté à la mesure du potentiel érosif d'un écoulement cavitant (Carrat et al., 2017). Lorsque des bulles de cavitation se développent sur un tel profil, leur implosion peut générer sur la paroi des pics de pression de courte durée $(\sim \mu \mathrm{s})$, de forte amplitude $(\sim \mathrm{GPa})$ localisés sur des zones de petite taille $(\sim \mu \mathrm{m})$. Ce phénomène qui peut être à l'origine d'endommagement par érosion de cavitation fait l'objet d'une attention particulière dans des applications industrielles telles que turbines, pompes, injecteurs de moteur diesel, etc. Il justifie le développement de ce type d'instrumentation instationnaire et requiert l'utilisation d'un dispositif d'acquisition haute fréquence.
Notre choix technique pour le réglage de l'incidence du profil s'est orienté vers un moteur brushless Kollmorgen équipé d'un résolveur relatif. Le pilotage de ce moteur se fait grâce à un variateur AKD par un protocole de communication Modbus TCP. Nous avons également choisi d'ajouter un frein de sécurité au moteur pour permettre de laisser le profil en incidence en désactivant le variateur.

Les paramètres de mise en incidence (angles, vitesses, profil temporel de variation d'incidence) sont chargés dans le variateur par l'intermédiaire de tâches réalisables par l'utilisateur. Le programme LabVIEW permet de réaliser ces tâches et d'imposer un mouvement au profil instrumenté. Ce mouvement peut être une mise en incidence simple avec désactivation du variateur par l'intermédiaire du frein de parking pour des essais en régime stationnaire à incidence fixe ou bien un déplacement temporel par profil imposé (sinus par exemple) permettant de réaliser des essais en régime instationnaire. La figure 4 montre la programmation et l'envoi des paramètres de mise en incidence du profil au variateur par le protocole ModBus TCP. Cette programmation consiste à ouvrir une communication avec le variateur par le biais d'une adresse IP, puis à charger un angle d'incidence pour le profil dans une tâche de mouvement choisi. Ensuite, la mise en mouvement du profil est réalisée. La programmation se termine en fermant la communication avec le variateur.

\section{L'ACQUISITION DES CAPTEURS HAUTE FRÉQUENCE}

Le profil piloté ci-dessus est instrumenté par une matrice de capteurs de pression PVDF (voir figure 3). L'acquisition des voies de cette matrice de capteurs est réalisée par la carte d'acquisition NI PXIe 5105 haute fréquence (jusqu'à $60 \mathrm{MHz}$ par voie) avec huit entrées simultanées dotées d'un échantillonneur bloqueur sur chaque voie. Cette carte est 


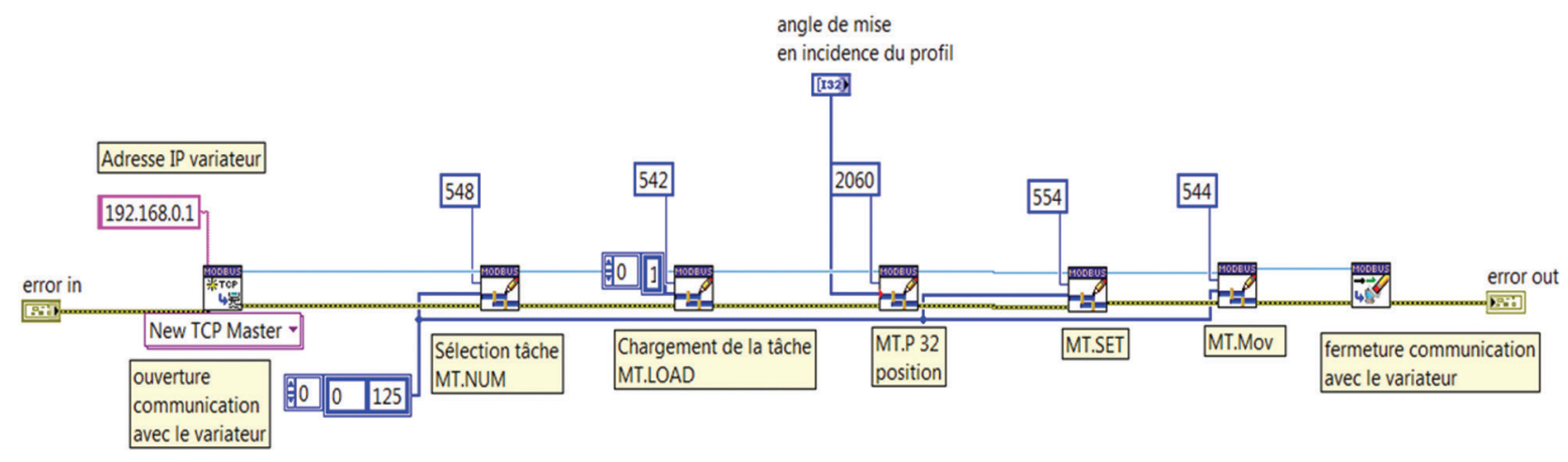

Figure 4 : Communication logicielle avec le variateur par le protocole ModBus TCP.

insérée dans le module PXI 1071. Un choix de matériel spécifique aux modules PXI a permis de garantir un taux de transfert de $1 \mathrm{GHz}$ entre le module d'acquisition et l'ordinateur. En parallèle, les bibliothèques de bas niveau pour l'accès aux fichiers d'enregistrement de données ont favorisé le maintien de ces performances au niveau de la programmation. L'acquisition des huit voies de pression, et donc des impacts des bulles de cavitation sur le profil, est réalisée en même temps que le déclenchement de la caméra par l'utilisateur et de l'enregistrement des données de supervision de la veine de cavitation. Les données sont stockées dans un fichier sous forme binaire pouvant être ouvert par un tableur ou Matlab.

\section{CONCLUSIONS}

Ces développements techniques permettent le contrôle commande d'un profil ainsi que les acquisitions des paramètres de la veine d'essai et celles des capteurs PVDF dans une même programmation. Ils offrent de nouveaux moyens pour l'étude des écoulements cavitants et permettront en particulier une meilleure évaluation du risque d'endommagement de profils hydrauliques par érosion de cavitation.

\section{REMERCIEMENTS}

Cette opération a bénéficié du soutien de GE dans le cadre de la chaire Hydro'Like gérée par la fondation G-INP.
Les auteurs remercient M. Yves Lecoffre pour sa contribution au design hydraulique de la nouvelle veine d'essais ainsi que M. Jean Bastien Carrat pour son étude sur les capteurs PVDF.

\section{RÉFÉRENCES}

Aumelas V., Lecoffre Y., Maj G., Franc J.-P. (2016) Micro-Bubbles Seeding For Flow Characterization. 28th Iahr Symposium On Hydraulic Machinery And Systems. July 4-8, 2016, Grenoble, France.

Aumelas V., Maj G., Franc J.-P., Boucheron R. (2018) Microbubbles Seeding For Optical Velocity Measurement (Ldv And Piv): An Experimental Validation. 19th International Symposium On Applications Of Laser And Imaging Techniques To Fluid Mechanics. July 16-19, 2018, Lisbon, Portugal.

Briançon-Marjollet L., Michel J.M. (1990) - The Hydrodynamic Tunnel of I.M.G.: Former and Recent Equipments. J. Fluids Eng, 112(3) : 338-342.

Carrat J.B., Fortes-Patella R., Franc J.-P. (2017) Assessment Of Cavitating Flow Aggressiveness On A Hydrofoil: Experimental And Numerical Approaches, Paper No. Fedsm2017-69187, Pp. V01at05a013, Asme. Fluids Engineering Division Summer Meeting, Hawaii, USA, July 30-August 3, 2017.

Guittet L., Kusulja M., Maitre T. (2004) - Mise en place d'un moyen d'essais pour les hydroliennes. Proceeding du Congrès Sea Tech Week sur les Energies Renouvelables en Mer, Brest, 18-22 octobre. 